\title{
A non-interventional multicenter study to document the implants success and survival rates in daily dental practices of the CONELOG screw-line implant
}

\author{
Claudio Cacaci $^{1} \cdot$ Karl-Ludwig Ackermann $^{2} \cdot$ Thomas Barth $^{3} \cdot$ Steffen Kistler $^{4} \cdot$ Michael Stiller $^{5} \cdot$ Markus Schlee $^{6}$ (D)
}

Received: 8 June 2017 / Accepted: 20 September 2018 / Published online: 15 October 2018

(C) The Author(s) 2018

\begin{abstract}
Objective This multicenter prospective non-interventional study evaluated the success and survival of the CONELOG implants inserted according to the standard protocol with one- or two-step surgery in daily practice three years after loading. Changes of soft tissue and bone level over time, esthetical outcome, and patient satisfaction were assessed.

Materials and methods The study included patients in six centers. The implants were used in accordance with standard practice of each particular center, and the use was documented systematically. Failure rate, implant success, bone level changes, different clinical parameters, and patients' satisfaction were assessed.

Results In total, 130 dental implants were placed in 94 patients (64 female, 30 male). Mean age of patients was 50.4 \pm 13.7 . At 3year post-loading, 108 implants in 78 patients were available for evaluation. Success and survival rate were $98.4 \%$ after placement and 100\% 36 months post-loading. Bone loss from surgery to loading was $0.52 \pm 0.55 \mathrm{~mm}$. From loading to $36 \mathrm{months}$ post-loading, the bone level change remained stable. Patient assessments were performed for function, ability to chew, speech, esthetics, and general satisfaction. Patients were very satisfied $(82.3 \%)$ or satisfied $(16.1 \%)$. None of the patients was dissatisfied. Conclusion The 3-year results of this non-interventional multicenter study indicate that the CONELOG implants are reliable and effective over the course of the observation while used in standard conditions of daily environment and confirm the results obtained in controlled clinical trials.

Clinical relevance Performance of CONELOG implants under daily routine is similar to controlled clinical trials.
\end{abstract}

Keywords Dental implant $\cdot$ Success rate $\cdot$ Life-table analysis $\cdot$ Daily dental practices $\cdot$ Conical connection

\section{Introduction}

The use of dental implants to restore partially or edentulous patients is very well documented since more than 30 years in systematic studies. Randomized controlled clinical trials

Markus Schlee

markus.schlee@32schoenezaehne.de

Munich, Germany

Filderstadt, Germany

Leipzig, Germany

Landsberg a. L., Germany

5 Berlin, Germany

6 Department of Maxillofacial Surgery, Goethe University Frankfurt, Bayreuther Strasse 39, 91301 Forchheim, Germany in a predefined environment report on the efficacy of the treatment. They include standard protocols and a welldefined number of inclusion and exclusion criteria selecting therefore the ideal patients. Osseointegrated implants have demonstrated long-term success in the rehabilitation of totally edentulous patients [1-3] as well as single or multiple teeth replacement as reported for the CAMLOG implant system [4, 5].

Implant success and survival rates have been measured in controlled clinical studies. These studies were criticized as not reflecting the real situation in dental practices. Investigators participating in formal clinical studies found themselves in a special situation, because often, the study protocol will give the investigators more time to treat patients as compared to the usual treatment protocols in daily dental practices. It can be argued that the results of clinical studies might be biased because of this special situation. These studies are also very well legitimated; however, the 
question if the offered treatment works under ordinary or daily circumstances, i.e., effectiveness, should be taken into account. Systematic longitudinal studies reflecting the regular use of implants treatment should be part of the systematic assessment of implants. The majority of the publications reported retrospective studies [6-8]. Few prospective clinical studies involving a large number of patients in daily dental practice are available. In a field trial involving more than 700 patients in 16 countries over a 5year period, the authors demonstrated the necessity of such studies to reinforce the results of formal clinical studies [9].

This non-interventional study was designed to provide data for life-table analysis regarding the performance of the CONELOG implants and the supra-construction under daily practices.

The primary objective was to analyze implants survival and success rates over 36 months post-loading for single or multiple tooth replacement in the maxilla or the mandible. Data were compared to the results of already published clinical studies and retrospective analyses. Secondary objectives included the evaluation of patients' satisfaction, changes of bone level over time, soft tissue parameters, and the esthetical outcome.

\section{Materials and methods}

\section{Study design and population}

The prospective multicenter non-interventional study was performed in accordance with the German medical devices law, the Declaration of Helsinki, and good clinical practice. The study protocol was approved by the international ethical committee of Freiburg (Germany, Ref. 010/1833). The reporting of this observational study conforms to the STROBE statement [10].

Six study centers in the German cities Berlin, Filderstadt, Forchheim, Landsberg a. L., Leipzig, and Munich participated in this study. Patients were recruited according to the study inclusion and exclusion criteria. Male and female patients had to be aged $\geq 18$ and one or several teeth missing in the maxilla or mandible. Sufficient crestal bone should be available at the implantation site as only small bone augmentations were allowed (e.g., autogenous bone harvested during drilling). The placed restoration had an opposing occlusion. Apart from the standard contra-indications for implant placement, heavy smokers ( $>10$ cigarettes or equivalent per day), pregnant and nursing women, socket preservation, and major augmentations less than 6 months before implant placement were excluded from the study. Daily patients requesting implant treatment were informed about the study and were included after signing a written informed consent.

\section{Material and surgical procedure}

The study included conical endosseous implant bodies with internal conical implant abutment connections (CONELOG SCREW-LINE implants; Camlog Biotechnologies AG, Basel, Switzerland) with diameters of $3.8 \mathrm{~mm}, 4.3 \mathrm{~mm}$, and $5.0 \mathrm{~mm}$, and lengths of $11 \mathrm{~mm}$ and $13 \mathrm{~mm}$, and their corresponding prosthetic components (platform switching). At surgery, implant placement was performed according to the manufacturer's recommendations and as usual in the individual study center. Implant diameter and length as well as level of implant insertion were selected in accordance with the patient indications. Surgery was performed under local anesthesia, and systematic antibiotics were given according to the standard procedure of each study center. To secure the postsurgical oral hygiene, the patients were advised to rinse with chlorhexidine or to gently brush the implant site until suture removal. For each patient, several parameters were documented. These included (1) type and number of implants, (2) implant site, (3) type of surgery, (4) bone quality according to Lekholm [11], (5) crestal ridge width and height, (6) distance implant shoulder to first visible bone contact at placement, and (7) primary implant stability (insertion torques or hand testing). Sutures were removed 7-14 days after implant placement.

The implants were allowed to heal submerged or transmucosal for at least 6 weeks based on the surgeons' clinical judgment, given indications, and patients' needs and preferences. At least 12 weeks were given, if a bone class IV was detected. Immediately after implant placement, intra-oral nonstandardized photographs and radiographs were taken. Suture removal and post-surgical care was done according to the study centers' routine practice. In addition, patients' complaints and quality of healing were assessed.

\section{Second stage procedure}

For a submerged healing, a second stage procedure was performed according to the standards of the study centers. Cylindrical or wide body gingiva formers were installed based on the surgeons' clinical judgment. Suture removal was done 7-14 days after second stage surgery according to the study centers' routine.

\section{Prosthesis placement}

The restoration protocol followed the standard time schedules as performed in the study centers. Implants were loaded with single crowns or fixed partial denture retained by a maximum of two implants. At prosthesis placement implant mobility, implant success [12] and survival, type of restoration, change of smoking and hygiene habits, Plaque Index (PI), and Sulcus Bleeding Index (SBI) were assessed. 
Patients were scheduled to follow-ups 6, 12, 24, and 36 months post-loading for the assessment of the study parameter. Depending on the investigators' standard postoperative protocol, follow-up appointments could be scheduled slightly differently. Non-standardized X-rays as usual in the individual centers and standardized photographs were taken. The oral health status was measured by assessing the PI and $\mathrm{SBI}$ if routinely performed in the practice.

\section{Patients' satisfaction}

Patients' reported outcome measures (PROMs) were assessed using a questionnaire including five items. The patient questionnaire addressed the (I) comfort, (II) appearance, (III) ability to chew, (IV) ability to taste, and (V) general satisfaction of the patient. The questionnaire was filled out at the final prosthesis loading as well as at follow-up appointments 6, 12, 24, and 36 months post-loading.

\section{Study outcomes}

The primary outcome was to assess implant survival and success of the implants 36 months post-loading with the final prosthesis. Secondary outcomes were changes of the bone level over time, evaluation of peri-implant soft tissue, and esthetical parameters as well as the evaluation of patients' satisfaction.

\section{Measurements}

Changes in crestal bone levels were assessed radiologically measuring the distance implant shoulder to first visible bone contact (DIB) at the mesial and distal site of an implant. All available radiographs were taken for evaluation, either periapical radiographs or OPTGs. The radiographs were not standardized. Non-digital radiographs were digitized by scanning (EPSON Perfection V700 Photo). The radiographs were analyzed using software ImageJ 1.44p (http://imagej.nih.gov/ij). Measurements at the mesial and distal sites were taken with an accuracy of $0.1 \mathrm{~mm}$ and averaged to obtain the bone level per implant. Changes of the bone level were calculated over the intervals "surgery to loading," as well as "loading to 12,24 , and 36 months post-loading." If radiographs were not available at one time point, changes in bone level were not analyzed for the implant and specified intervals.

Different soft tissue parameters were assessed over time. Parameters included PI and SBI at four points (buccal, lingual, distal, and mesial sites) on each implant [13]. An attached gingiva was determined by the presence or absence on midlabial and mid-lingual surfaces. The modified plaque index (MPI) was determined on a scale of 0 to 3 with $0=$ no plaque, 1 = separate flecks of plaque at the cervical margin, 2 = plaque can be seen by eye, and 3 = abundance of soft matter. The lower the number the less plaque is present on the tooth.

\section{Adverse events}

General health condition of the patients at the time of implantation was assessed using the ASA classification system [14], and adverse events (AE) were documented throughout the study.

\section{Statistical analysis}

Descriptive statistics were performed with IBM SPSS V 19.0 (IBM Corp., Armonk, NY, USA). The study was designed to provide data for life-table analysis regarding the performance of the implants when restored after a healing period of at least 6 weeks. The data were analyzed for implant success and survival rates after 1 year and yearly thereafter. All safety aspects of the implant treatment were documented and analyzed. Loading with the final prosthesis was set as baseline for all analysis.

\section{Results}

A total of 130 implants in 94 patients were placed by the 6 study centers (Table 1). Distribution of patients and placed implants was homogenous. Thirty patients were male (31.9\%), 64 female (68.1\%). The mean age at the time of implantation was $50.4 \pm 13.7$ (19.1 to 75.6 years). In 86 patients, normal health conditions ASA 1 (91.5\%) were observed, while eight patients were classified as ASA 2 $(8.5 \%)$. Single implant was inserted in 62 patients $(66.0 \%)$ and 2 implants in 28 patients $(29.8 \%)$, and 4 patients $(4.3 \%)$ received three implants. Antibiotic prophylaxis was given to 67 patients $(71.3 \%)$, while 27 patients $(28.7 \%)$ were not treated and 14 patients (14.9\%) smoked up to 10 cigarettes/day.

Tooth extraction was necessary mainly because of endodontic failure $(41.5 \%)$. Other reasons were fractures $(13.1 \%)$, periodontal reasons $(18.5 \%)$, caries $(3.8 \%)$, and others $(23.1 \%)$. A total of 126 implants $(96.9 \%)$ were placed on healed extraction sites, while 4 implants were placed immediately after tooth extraction (3.1\%). Time between tooth extraction and implant placement was $3.06 \pm 6.37$ years on average. Before implantation, the crest in 18 implantation sites was flattened (13.8\%), while 112 implantation sites $(86.2 \%)$ were left untreated. Immediately after implant placement, all 130 implants showed primary stability. For 116 implants, a measurement of the insertion depth was taken from the radiographs: 10 implants were placed supracrestally, 56 implants equicrestally, and 50 implants subcrestally. The mean insertion depth was $0.32 \pm 0.53 \mathrm{~mm}$ ( $\mathrm{min},-1.75 \mathrm{~mm}$; max, $0.55 \mathrm{~mm}$ ) subcrestally. Tooth-implant distance was on average 
Table 1 Demographic and clinical parameter of the study population and the implanted sites

\begin{tabular}{ll}
\hline Patients/implants & $94 / 130$ \\
Center 1 & $15 / 19$ \\
Center 2 & $9 / 17$ \\
Center 3 & $18 / 26$ \\
Center 4 & $14 / 16$ \\
Center 5 & $18 / 26$ \\
Center 6 & $20 / 26$ \\
Mean age \pm SD (years) & $50.4 \pm 13.7$ \\
Gender male/female & $30 / 64$ \\
Implants per patient & \\
1 implant & 62 \\
2 implants & 28 \\
3 implants & 4 \\
Tobacco use & \\
Never use tobacco & 64 \\
Stopped tobacco use & 16 \\
Less than 10 cigarettes/day & 14 \\
Bone quality; $n$ implants $(\%)$ & \\
Class I & $13(10.0)$ \\
Class II & $67(51.5)$ \\
Class III & $43(33.1)$ \\
Class IV & $7(5.4)$ \\
Time of implantation; $n$ implants $(\%)$ & \\
Immediate implantation & $4(3.1)$ \\
Delayed implantation & $126(96.9)$ \\
Type of healing; $n$ implants $(\%)$ & \\
Submerged (2-stage) & \\
Transgingival (1-stage) & \\
\hline & \\
&
\end{tabular}

$3.46 \pm 1.63 \mathrm{~mm}$ mesial and $3.48 \pm 1.44 \mathrm{~mm}$ distal. The number of implants per position is shown in Table 2 and the diameter and height of the implants in Table 3 . The majority of the implant sites presented a D2 $(n=67 ; 51.5 \%)$ or D3 $(n=$ $43 ; 33.1 \%$ ) bone type. The remaining 20 sites were classified as either $\mathrm{D} 1(n=13 ; 10.0 \%)$ or $\mathrm{D} 4(n=7 ; 5.4 \%)$.

Submerged healing was applied in $66.7 \%$ of all implants, transgingival healing in $33.3 \%$. During healing, two patients showed minor wound dehiscence which healed uneventfully. Prosthetic information was available for 122 implants in 89 patients. Three patients with 4 implants were not loaded due to

Table 2 Overview of the implants placed. Number and position of implants and distribution of implants according to FDI tooth positions

$\begin{array}{llllllllllllllll}0 & 0 & 6 & 10 & 6 & 2 & 5 & 6 & 4 & 4 & 1 & 3 & 6 & 5 & 1 & 0\end{array}$

$\begin{array}{llllllllllllllll}18 & 17 & 16 & 15 & 14 & 13 & 12 & 11 & 21 & 22 & 23 & 24 & 25 & 26 & 27 & 28\end{array}$

$\begin{array}{llllllllllllllll}48 & 47 & 46 & 45 & 44 & 43 & 42 & 41 & 31 & 32 & 33 & 34 & 35 & 36 & 37 & 38\end{array}$

$\begin{array}{llllllllllllllll}0 & 2 & 19 & 6 & 2 & 0 & 0 & 0 & 0 & 0 & 0 & 1 & 9 & 26 & 6 & 0\end{array}$
Table 3 Overview of the implants placed. Implant diameter and length and implant distribution according to length and diameter. $37.7 \%$ of the implants were of $\varnothing$ $3.8 \mathrm{~mm}, 61.5 \%$ of $\varnothing$ $4.3 \mathrm{~mm}$

\begin{tabular}{|c|c|c|c|}
\hline \multirow[t]{2}{*}{ Diameter } & \multicolumn{2}{|l|}{ Length } & \multirow[t]{2}{*}{ Total } \\
\hline & $11 \mathrm{~mm}$ & $13 \mathrm{~mm}$ & \\
\hline Ø $3.8 \mathrm{~mm}$ & 28 & 21 & 49 \\
\hline$\varnothing 4.3 \mathrm{~mm}$ & 40 & 40 & 80 \\
\hline$\varnothing 5.0 \mathrm{~mm}$ & 1 & 0 & 1 \\
\hline Total & 69 & 61 & 130 \\
\hline
\end{tabular}

financial issues, one patient withdrew consent, and two implants had to be extracted. Implants were loaded $22.6 \pm$ 12.5 weeks (min, 8.1 weeks; max, 79.1 weeks) post-implantation. One hundred three implants were loaded with a single crown, while a fixed partial denture was used in 9 cases (16 implants). In one patient, a bridge was placed. No detailed loading information was available for another patient. Fullceramic restorations were placed in 62 cases (54.9\%) and ceramo-metal restorations in 51 cases $(45.1 \%)$. The restorations were either screw-retained $(18.6 \%)$ or cementretained $(81.4 \%)$ with the following materials: TempBond ( 25 cases; Kerr GmbH, Germany), Harvard cement ( 7 cases; Harvard Dental International GmbH, Germany), KetacTM Cem (3M ESPE, Germany) and Freegenol (GC Europe, Belgium) in one case each, Durelon (45 cases; 3M ESPE, Germany), Implant Link (7 cases; Detax GmbH, Germany), or other ( 6 cases). The mean crown to implant ratio was 0.82 \pm 0.23 ( $\mathrm{min}, 0.42$ to 1.62 ). The final prosthesis was placed in 58 cases in functional occlusion $(51.3 \%)$, in 48 cases in full occlusion $(42.5 \%)$, in six cases out of occlusion $(5.3 \%)$, or other $(0.9 \%)$.

Up to 36 months post-loading, further 4 patients ( 5 implants) withdrew consent, 6 patients ( 8 implants) were lost to followup, and one patient (1 implant) died. Hence, 36 months postloading, 108 implants in 78 patients were considered for evaluation.

\section{Implant survival and success}

Two early failures were observed in the mandible. One implant was extracted due to infection in a smoker 2 months after surgery, one implant was extracted due to radiolucency five and a half months after surgery. In both cases, delayed implant placement and submerged healing were applied. The life-table analysis and survival rate respectively were calculated from the date of the implantation as well as from baseline. The last patient had the 36 months post-loading follow-up 53 months postsurgery due to "delayed" loading. The success and survival rate were the same scoring with $98.4 \%$ after 36 months postloading starting from implantation. Post-loading, no additional complication and implant loss was reported. Therefore, the success and survival rate was $100 \%$ from implant loading to 36 months follow-up according to the protocol. 


\section{Bone level changes}

Table 4 presents the mean bone level changes of the implants from surgery to 36 months post-loading. Bone remodeling around the implant was noticeable from surgery to loading, presenting a mean value of $-0.52 \pm 0.55 \mathrm{~mm}$. From loading to the 36 months follow-up, the mean change in crestal bone remained very stable $(-0.04 \pm 0.40 \mathrm{~mm})$. Split into three groups, $37.8 \%$ of the implants were noted with a bone gain up to $1.0 \mathrm{~mm}$ (mean $0.34 \pm 0.21 \mathrm{~mm}$ ), $25.6 \%$ of the implants revealed no change in bone level, and $36.7 \%$ of the implants experienced bone loss (mean $-0.44 \pm 0.30 \mathrm{~mm}$ ). The remodeling event was descriptively evaluated depending from the different insertion depths of the implants. Implants placed equicrestally presented mean bone level changes from surgery to loading of $-0.34 \pm 0.42 \mathrm{~mm}$ superior to implants placed subcrestally showing mean bone level changes of $-0.73 \pm$ $0.62 \mathrm{~mm}$. Superiority of the equicrestal group remained until the last follow-up (surgery to 36 months post-loading: equicrestal $-0.27 \pm 0.53 \mathrm{~mm}$ versus subcrestal $-0.79 \pm$ $0.53 \mathrm{~mm})$.

\section{Evaluation of soft tissue parameter}

The MPI was $0.08 \pm 0.29$ at time of loading and $0.20 \pm 0.40$ 36 months post-loading, which represents a well-maintained entity of patients (Table 5). Values correspond with SBI 0.10 \pm 0.39 at time of loading and $0.08 \pm 0.1936$ months postloading (Table 5). Attached gingiva was assessed to be present around $95.5 \%$ mid-labial and $94.6 \%$ mid-lingual all implants. The gingiva was attached of all implants (100\%) 36 months after prosthesis loading.

\section{Oral hygiene}

Oral hygiene status is shown in Fig. 1. Before implantation, patients presented an excellent (31.9\%), good (66.0\%), and fair oral hygiene $(2.1 \%)$. At the last follow-up 36 months post-loading, patients presented an excellent (30.7\%), good (56.0\%), and fair (12.0\%) oral hygiene. At the 6- and 36month follow-up, a poor oral hygiene was documented for one patient.

Table 4 Mean crestal bone level changes in $\mathrm{mm}$

\begin{tabular}{lrl}
\hline & $N$ & Mean \pm SD (mm) \\
\hline Surgery to loading & 103 & $-0.52 \pm 0.55$ \\
Loading to 12-month & 93 & $-0.04 \pm 0.37$ \\
Loading to 24-month & 79 & $-0.04 \pm 0.41$ \\
Loading to 36-month & 90 & $-0.04 \pm 0.40$ \\
\hline
\end{tabular}

Table 5 Soft-tissue health: Plaque Index (PI); Sulcus Bleeding Index (SBI)

\begin{tabular}{llllll}
\hline & \multicolumn{2}{c}{ Plaque index (score 0-3) } & & \multicolumn{2}{l}{ Sulcus bleeding index (score 0-3) } \\
\cline { 2 - 3 } \cline { 5 - 6 } & $N$ & Mean $\pm \mathrm{SD}$ & & $N$ & Mean $\pm \mathrm{SD}$ \\
\hline Loading & 80 & $0.08 \pm 0.29$ & & 81 & $0.10 \pm 0.39$ \\
12-month & 97 & $0.18 \pm 0.42$ & & 96 & $0.08 \pm 0.24$ \\
24-month & 80 & $0.11 \pm 0.27$ & & 82 & $0.11 \pm 0.29$ \\
36-month & 90 & $0.20 \pm 0.40$ & & 89 & $0.08 \pm 0.19$ \\
\hline
\end{tabular}

\section{Patient-reported outcome measures}

As part of the non-interventional multicenter study, patients rated the functional and esthetic outcome of the dental restoration. At the last follow-up 36 months post-loading, on a category scale of 1 (maximal satisfied) to 5 (very unsatisfied), $82.3 \%$ of the patients were maximal satisfied, while $16.1 \%$ scored with satisfied (Fig. 2). 1.6\% of the patients scored with middle. One patient was unsatisfied with the appearance due to esthetic problems because of peri-implant soft tissue recession.

\section{Discussion}

The replacement of missing teeth with endosseous dental implants is a commonly accepted treatment procedure. Prosthetic teeth are attached to the implants to restore masticatory function. Longitudinal studies have shown that implants and prostheses can work effectively for more than 20 years [3]. In recent years, patients' needs have increased in terms of the esthetic and functional outcome of the dental restoration. For CONELOG implants as well as for the implant surface, controlled clinical trials and retrospective analyses reported high survival and success rates as well as good functional performances $[4,15,16]$. However, in general, it could be criticized that results of controlled clinical studies do not reflect the real situation in daily dental practices. Thus, the primary objective of this non-interventional multicenter study was to estimate data for life-table analysis of the survival of CONELOG implants under daily practice conditions. The advantage of the present study was to evaluate the performance of these implants under conditions which reflect those seen in daily dental practices. According to the study protocol, minimal exclusion criteria were applied in the selection of study participants alongside the usual contraindications and the technical procedure was not standardized. As a result, study participants recruited were heterogeneous as typically seen in daily dental practices. After an observation period of 36 months, the implants demonstrated good performance with respect to implant survival and success. From the 130 implants placed, only 2 
Fig. 1 Oral hygiene of patients over time. Oral hygiene was assessed and revised with the patient at pre-surgery, postsurgery, loading, and 6-, 12-, 24-, and 36-month post-loading. $n=$ 69-95

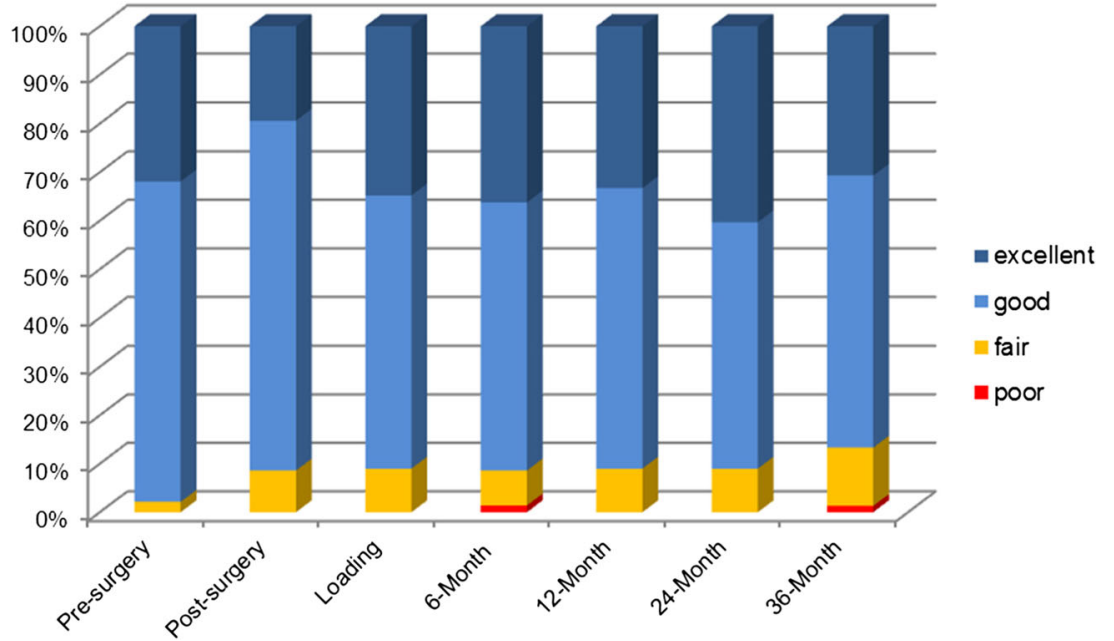

implants were lost resulting in survival and success rates of 98.4\% from surgery to loading. Post-loading, no additional implant had to be explanted. Thus, although the selection of study participants was less-stringent, the success and survival rates of implants and their corresponding prosthetic components in daily dental practices were similar to the results of controlled clinical trials.

Changes in crestal bone level are well documented in the literature. Bone remodeling is reported to take place between surgery and loading. The reported changes are generally around $0.5 \mathrm{~mm}[15,17-19]$ but can reach more than $1 \mathrm{~mm}$ [20]. The present study is well in accordance with the findings with a bone remodeling of $-0.52 \pm 0.55 \mathrm{~mm}$.

From loading to the 36 months follow-up, stable crestal bone levels at the implant shoulder were documented ($0.04 \pm 0.40 \mathrm{~mm}$ ). Interestingly, at $63 \%$ of the implants, no change in bone level or even bone gain was observed postloading. This appears to reflect the results of a controlled clinical study published recently by Moergel et al. $[15,21]$. The authors demonstrated similar bone remodeling at the implant shoulder and good preservation of the crestal bone (+
$0.12 \mathrm{~mm} \pm 0.42 \mathrm{~mm}$ ) in the mandible 1 year after implant loading. The integrated platform switching of the implants might additionally contribute to the stabilization of the bone as reported in several studies [18, 19, 22].

In the case of the present study, the differences in bone level changes over time between equicrestal- and subcrestalplaced implants were evaluated. Between surgery and loading, implants placed subcrestally presented major mean bone loss of $-0.73 \pm 0.62 \mathrm{~mm}$ compared to that of the equicrestal group, presenting a bone loss of only $-0.34 \pm 0.42 \mathrm{~mm}$. The increased bone loss of implants placed subcrestally is in accordance with other clinical studies published [15, 23] reporting similar bone remodeling between surgery and loading, particularly for the implants placed subcrestally are in accordance with the consensus report published by Schwarz et al. [24]. Taken together, our findings on bone remodeling after implantation mirror previously published reports highlighting the excellent performance of CONELOG implants in dental implant therapy in daily dental practices.

PROMs have been reported in several studies and represent a well-described non-invasive measurement; however they

\section{2-month post-loading}

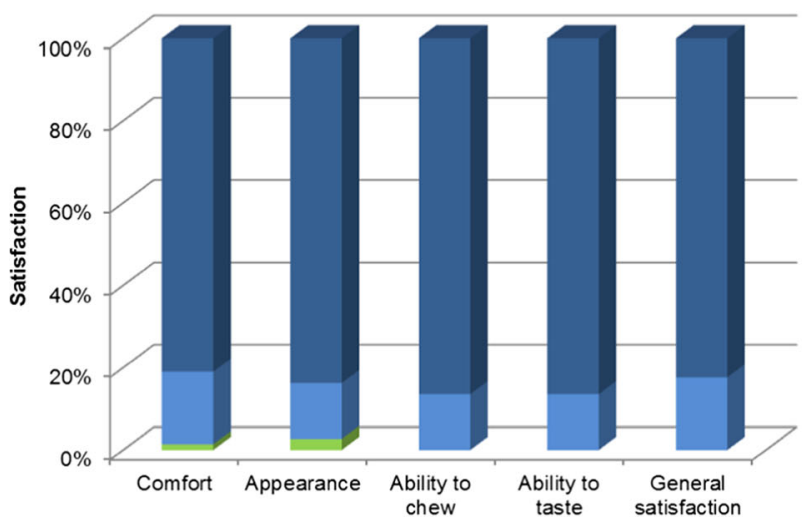

Fig. 2 Patients' satisfaction over time. Patients' satisfaction was assessed at loading and 12-, 24-, and 36-month post-loading using a patients'

\section{6-month post-loading}

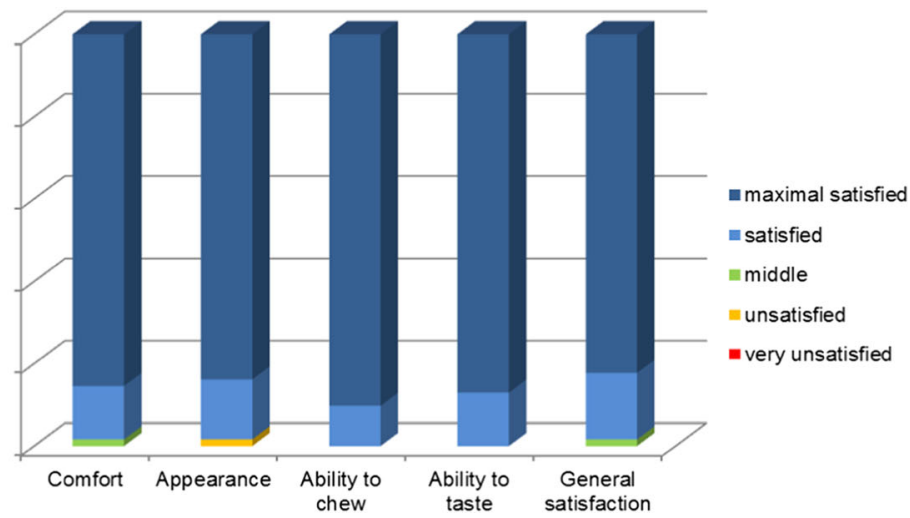

questionnaire. A vote was possible on a scale of 1 (very unsatisfied) to 5 (maximal satisfied). $n=48-74$ 
might present a lack of standardization $[25,26]$. Patients' satisfaction including esthetical and functional parameters as well as the oral hygiene status was evaluated. Although objective judgements using reproducible classification systems try to describe the esthetic outcome in implant dentistry, esthetics are subjective. Parameters considered to be important by the investigator might not be reliable with patients' subjective satisfaction about the functional and esthetic results [27]. Patients' satisfaction can be an important indicator for measuring the overall success of the dental restoration. The data of the present study revealed that $82.3 \%$ of the patients were maximal satisfied and $16.1 \%$ satisfied at the 36 months follow-up appointment. These very satisfying results might result from the excellent compliance of the patients in terms of oral hygiene. They are in accordance with the results published by others [28-30].

In summary, non-interventional studies may substantially reduce the possibility of bias due to the increased heterogeneity of study participants. Within a non-interventional study, it is difficult to standardize procedures among study centers. Different treatment protocols were applied beginning with the type of implantation (immediate versus delayed implantation), the healing procedure (submerged or transgingival healing), and the prosthetic restorations (screw- versus cement-retained; single crowns and FPDs). However, several studies and systematic reviews showed high survival and success rates as well as excellent clinical conditions without statistically significant differences between the applied treatment types [31-35]. Most implants in the present study were placed in healed sites (97\%), healed submerged (67\%), and were restored with cement retained ( $81 \%$ ) single crown restorations $(85 \%)$. The study results confirm that the surgical and restorative techniques have few to no impact on the outcome (survival and success). Within the non-interventional setting, study participants might be more difficult to follow-up. Patients who are satisfied with their restorations and without any complications might tend to omit the control visits. In this study, $17 \%$ of the initially treated patients withdrew from the study or could not be followed up for different reasons. This is in accordance with dropout rates of other clinical field trials [9]. Despite these possible limitations, non-interventional studies have great potential, evaluating dental treatment methods in daily dental practice reducing the risk of bias toward more favorable outcomes that may occur in controlled clinical studies.

\section{Conclusion}

The present non-interventional study demonstrated successful functional and esthetic outcomes of CONELOG implants. Upon loading, crestal bone levels remained stable (26\%), and for almost $38 \%$ of the implants, bone gain was documented post-loading. Thus, results presented here mirror the outcome of already published controlled clinical studies and retrospective analyses confirming CONELOG implants clinical appropriateness in daily dental practices. Noninterventional studies have the potential to complement the results achieved in controlled clinical studies and to assess the performance of dental implants in daily dental practice.

Funding This study was funded by an unrestricted grant of the Oral Reconstruction Foundation (previously CAMLOG Foundation/Grant NISCAM01/10). Study monitoring and data analysis were supported by the Oral Reconstruction Foundation.

\section{Compliance with ethical standards}

Conflict of interest The authors declare that they have no conflict of interest. All authors lecture for the Camlog company.

Ethical approval All procedures performed in studies involving human participants were in accordance with the ethical standards of the institutional and/or national research committee and with the 1964 Helsinki declaration and its later amendments or comparable ethical standards.

Informed consent Informed consent was obtained from all individual participants included in the study.

Open Access This article is distributed under the terms of the Creative Commons Attribution 4.0 International License (http:// creativecommons.org/licenses/by/4.0/), which permits unrestricted use, distribution, and reproduction in any medium, provided you give appropriate credit to the original author(s) and the source, provide a link to the Creative Commons license, and indicate if changes were made.

\section{References}

1. Branemark PI, Hansson BO, Adell R, Breine U, Lindström J, Hallén O, Ohman A (1977) Osseointegrated implants in the treatment of the edentulous jaw. Experience from a 10-year period. Scand J Plast Reconstr Surg Suppl 16:1-132

2. Hansson HA, Albrektsson T, Branemark PI (1983) Structural aspects of the interface between tissue and titanium implants. J Prosthet Dent 50(1):108-113

3. Krennmair G, Weinländer M, Krainhöfner M, Piehslinger E (2006) Implant-supported mandibular overdentures retained with ball or telescopic crown attachments: a 3-year prospective study. Int J Prosthodont 19(2):164-170

4. Strietzel FP, Reichart PA (2007) Oral rehabilitation using Camlog screw-cylinder implants with a particle-blasted and acid-etched microstructured surface. Results from a prospective study with special consideration of short implants. Clin Oral Implants Res 18(5): 591-600

5. Krennmair G, Krainhöfner M, Piehslinger E (2008) The influence of bar design (round versus milled bar) on prosthodontic maintenance of mandibular overdentures supported by 4 implants: a 5year prospective study. Int J Prosthodont 21(6):514-520

6. Eghbali A, De Bruyn H, De Rouck T, Cleymaet R, Wyn I, Cosyn J (2012) Single implant treatment in healing versus healed sites of the anterior maxilla: a clinical and radiographic evaluation. Clin Implant Dent Relat Res 14(3):336-346 
7. French D, Larjava H, Ofec R (2015) Retrospective cohort study of 4591 Straumann implants in private practice setting, with up to 10year follow-up. Part 1: multivariate survival analysis. Clin Oral Implants Res 26(11):1345-1354

8. Cosyn J, Raes S, De Meyer S, Raes F, Buyl R, Coomans D, De Bruyn H (2012) An analysis of the decision-making process for single implant treatment in general practice. J Clin Periodontol 39(2):166-172

9. Cochran D, Oates T, Morton D, Jones A, Buser D, Peters F (2007) Clinical field trial examining an implant with a sand-blasted, acidetched surface. J Periodontol 78(6):974-982

10. von Elm E, Altman DG, Egger M, Pocock SJ, Gøtzsche PC, Vandenbroucke JP (2008) STROBE initiative. The Strengthening the Reporting of Observational Studies in Epidemiology (STROBE) statement: guidelines for reporting observational studies. J Clin Epidemiol 61(4):344-349

11. Brånemark PI, Zarb GA, Albrektsson T (1986) Tissue-Integrated Prostheses: Osseointegration in Clinical Dentistry. Quintessence Publishing Vol. 2nd

12. Buser D, Ingimarsson S, Dula K, Lussi A, Hirt HP, Belser UC (2002) Long-term stability of osseointegrated implants in augmented bone: a 5-year prospective study in partially edentulous patients. Int J Periodontics Restorative Dent 22:109-117

13. Mombelli A, van Oosten MA, Schurch E Jr, Lang NP (1987) The microbiota associated with successful or failing osseointegrated titanium implants. Oral Microbiol Immunol 2(4):145-151

14. (ASA), A.S.o.A. ASA PHYSICAL STATUS CLASSIFICATION SYSTEM. . Last updated and approved by the ASA House of Delegates on October 15, 2014; Available from: https://www. asahq.org/resources/clinical-information/asa-physical-statusclassification-system.

15. Moergel M, Rocha S, Messias A, Nicolau P, Guerra F, Wagner W (2015) Radiographic evaluation of conical tapered platform-switched implants in the posterior mandible: 1-year results of a two-center prospective study. Clin Oral Implants Res 27(6):686-693

16. Semper W, Hildebrand D, Özyuvaci H, Nelson K (2007) Erfolgsrate von Implantaten mit sandgestrahlter und geätzter Oberfläche im Oberkiefer nach einer Einheilzeit von 12 Wochen: Eine retrospektive Analyse. Z Zahnärztl Impl 23(3):176-188

17. Wallkamm B, Ciocco M, Ettlin D, Syfrig B, Abbott W, Listrom R, Levin BP, Rosen PS (2015) Three-year outcomes of Straumann Bone Level SLActive dental implants in daily dental practice: a prospective non-interventional study. Quintessence Int 46(7):591-602

18. Guerra F, Wagner W, Wiltfang J, Rocha S, Moergel M, Behrens E, Nicolau P (2014) Platform switch versus platform match in the posterior mandible - 1-year results of a multicenter randomized clinical trial. J Clin Periodontol 41:521-529

19. Rocha S, Wagner W, Wiltfang J, Nicolau P, Moergel M, Messias A, Behrens E, Guerra F (2016) Effect of platform switching on crestal bone levels around implants in the posterior mandible: 3 years results from a multicentre randomized clinical trial. J Clin Periodontol 43(4):374-382

20. Ravald N, Dahlgren S, Teiwik A, Gröndahl K (2013) Long-term evaluation of Astra Tech and Brånemark implants in patients treated with full-arch bridges. Results after 12-15 years. Clin Oral Implants Res 24(10):1144-1151

21. W. Wagner, M. Moergel, P. Nicolau, A. Messias, S. Rocha, F. Guerra (2015) Clinical performance of conical tapered and platform-switched implants in the posterior mandible - preliminary three year results of a prospective two-center study. P369 poster at EAO congress in Stockholm
22. Strietzel FP, Neumann K, Hertel M (2015) Impact of platform switching on marginal peri-implant bone-level changes. A systematic review and meta-analysis. Clin Oral Implants Res 26:342-358

23. Jung RE, Jones AA, Higginbottom FL, Wilson TG, Schoolfield J, Buser D, Hämmerle CH, Cochran DL (2008) The influence of nonmatching implant and abutment diameters on radiographic crestal bone levels in dogs. J Periodontol 79(2):260-270

24. Schwarz F,AlcoforadoG,Nelson K, Schaer A, Taylor T, Beuer F, Strietzel FP (2014) Impact of implant-abutment connection, positioning of the machined collar/microgap, and platform switching on crestal bone level changes . Camlog Foundation Consensus Report. ClinOral Implants Res 25:1301-1303

25. De Bruyn H, Raes S, Matthys C, Cosyn J (2015) The current use of patient-centered/reported outcomes in implant dentistry: a systematic review. Clin Oral Implants Res 26(Suppl.11):45-56

26. McGrath C, Lam O, Lang N (2012) An evidence-based review of patient-reported outcome measures in dental implant research among dentate subjects. J Clin Periodontol 39(Suppl.12):193-201

27. Chang M, Odman PA, Wennström JL, Andersson B (1999) Esthetic outcome of implant-supported single-tooth replacements assessed by the patient and by prosthodontists. Int J Prosthodont 12(4):335-341

28. Molina A, Sanz-Sánchez I, Martín C, Blanco J, Sanz M (2016) The effect of one-time abutment placement on interproximal bone levels and peri-implant soft tissues: a prospective randomized clinical trial. Clin Oral Implants Res:25. https://doi.org/10.1111/clr.12818 [Epub ahead of print]

29. Hämmerle CH, Jung RE, Sanz M, Chen S, Martin WC, Jackowski J, Multicenter study group (2012) Submerged and transmucosal healing yield the same clinical outcomes with two-piece implants in the anterior maxilla and mandible: interim 1-year results of a randomized, controlled clinical trial. Clin Oral Implants Res 23(2):211-219

30. Cochran DL, Jackson JM, Jones AA, Jones JD, Kaiser DA, Taylor TD, Weber HP, Higginbottom FL, Richardson JR, Oates T (2011) A 5-year prospective multicenter clinical trial of non-submerged dental implants with a titanium plasma-sprayed surface in 200 patients. J Periodontol 82(7):990-999

31. Esposito M, Grusovin MG, Chew YS, Coulthard P, Worthington HV (2009) One-stage versus two-stage implant placement. A Cochrane systematic review of randomised controlled clinical trials. Eur J Oral Implantol 2(2):91-99

32. Sanz M, Ivanoff CJ, Weingart D, Wiltfang J, Gahlert M, Cordaro L, Ganeles J, Bragger U, Jackowski J, Martin WC, Jung RE, Chen S, Hammerle C (2015) Clinical and radiologic outcomes after submerged and transmucosal implant placement with two-piece implants in the anterior maxilla and mandible: 3-year results of a randomized controlled clinical trial. Clin Implant Dent Relat Res 17(2):234-246

33. Esposito M, Grusovin MG, Polyzos IP, Felice P, Worthington HV (2010) Timing of implant placement after tooth extraction: immediate, immediate-delayed or delayed implants? A Cochrane systematic review. Eur J Oral Implantol 3(3):189-205

34. Sailer I, Mühlemann S, Zwahlen M, Hämmerle CH, Schneider D (2012) Cemented and screw-retained implant reconstructions: a systematic review of the survival and complication rates. Clin Oral Implants Res 23(Suppl 6):163-201

35. Muddugangadhar BC, Amarnath GS, Sonika R, Chheda PS, Gart A (2015) Meta-analysis of failure and survival rate of implantsupported single crowns, fixed partial denture and implant toothsupported prostheses. J Int Oral Health 7(9):11-17 